\title{
A Knowledge Infrastructure for the Dutch Immigration Office
}

\author{
Ronald Heller, Freek van Teeseling, and Menno Gülpers \\ Be Informed, Linie 620, 7325 DZ Apeldoorn, Netherlands \\ \{r.heller, f.vanteeseling, m.gulpers\} @beinformed.nl
}

\begin{abstract}
The Dutch Immigration Office is replacing its existing paper based case system with a fully electronic system with integrated decision support based on ontologies. The new award winning architecture (Dutch Architecture award 2009) is based on the principle of separation of concerns: data, knowledge and process. The architecture of the application, but especially the architecture of the knowledge models for decision and process support, is explained and shown in the demonstration.
\end{abstract}

Keywords: Knowledge models, paper based, case system, decision and process support, Be Informed, Run - Model - Analyze principle, knowledge as a service, separate know from flow.

\section{Background and Application Context}

The Dutch Immigration Office [4] decided to completely replace its existing (mostly) paper based case system and some form of decision support (decision trees). The new application had to be a completely electronic case system with integrated decision and process support, including a directly connected front office [5]. The case system is mainly targeted at the internal knowledge worker who handles the incoming requests. The front office is for clients (foreigners) who apply for a permit to stay in the Netherlands. This application replaces the current operational cluster of systems, that do not provide the flexibility and agility that is needed today. Management costs are way too high and changes take far too long to be implemented.

Ordina and Accenture together with Be Informed came up with the award winning architecture (Dutch Architecture Award 2009) [6] that is all about separation of concerns: data, knowledge and process. This enables and ensures the flexibility and agility that is so needed. When these concerns are not interconnected, the changes can be implemented, reviewed and validated in isolation, which shortens time needed for implementations as a whole, and thus enable greater agility. The semantic based knowledge support consists of a large set of models which serves both the front office (website [5]) and the back office (using SOA [2]). This implies that the same models are used for different audiences. The website offers functionality to decide what application to fill in, including tailor-made online forms, and the back office provides a range of services to support the case management system with e.g. process flows, decision support and generation of documents. The webportal is fully operational, 
whereas the back office components are currently being deployed in the organization. A similar architecture has been used in several other recent projects.

\section{Technology}

Be Informed is a software product to support complex and knowledge-intensive business processes. Using Be Informed software, organizations improve their service to customers and partners, streamline their working processes and achieve substantial gains in efficiency by delivering the appropriate knowledge in a direct and contextspecific manner to business users and customers. Be Informed gives organizations the ability to quickly adapt to changes in legislation or their surroundings.

Be Informed is an integrated platform for all required services, processes and tasks. This enables business partners to implement knowledge infrastructures in (large) enterprises, that can be used to build multi-product, multi-label and multi-lingual knowledge intensive applications, to address the challenges of a modern organization.

Be informed uses its own strongly typed syntax, mainly because the existing web standards [1] like OWL [7] and $\mathrm{RDF}(\mathrm{S})$ [8] have at least 2 major drawbacks in a deployed application like the one subject of this demonstration. First, there is a strong need to link textual and visual content to the models, to be displayed in certain parts of the application. Secondly, decisions and classifications can be modeled much more easily using a "Closed World" assumption rather than the usual "Open World" assumption.

In Be Informed Modeling, all models are based on a metamodel: the knowledge architecture[9]. It defines conceptual areas in the domain(s) and specifies their dependencies. It also defines the context of knowledge in surrounding IT systems, the organization's generic process and case systems, which are also part of Be informed.

Based on ontology design patterns [10], we divided the semantic models for the Dutch Immigration Office in 4 main areas:

- Core Taxonomies. Basic vocabulary of the organization (permits, actions, purpose of stay, criteria etc) which is referenced by many knowledge models.

- Regulations. For each set of regulations a semantic model is built to represent the applicable laws and regulations, and act as decision support in the operational process.

- Online front office. This is the portal for the customer (foreigner) to explore the information, get personalized advice and apply for a permit.

- Catalog. This is a documentation model serving as an explanatory model "about the other models" to help developers, modelers and business users to find their way in modeling.

Note that there is a loosely coupled $5^{\text {th }}$ area, that of a cross-governmental portal, leading foreigners coming to the Netherlands to all relevant governmental organizations, including the IND: "New to Holland" [3].

In the models we use strongly typed relations (instance, subclass, requires, depends on, etc.) connecting different concept types. In the metamodel concept types are defined for Control data, Actions, Conditions for actions, States, Roles and Generic Properties. 


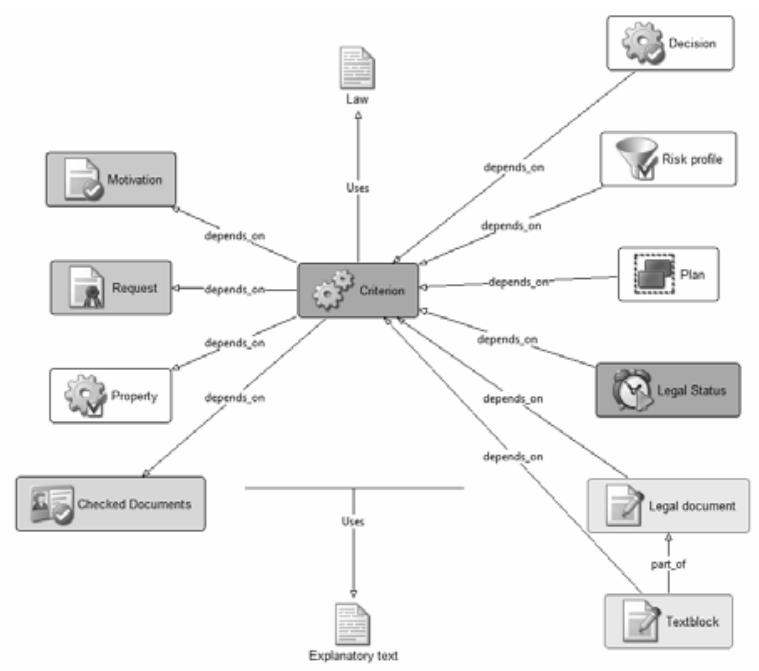

Fig. 1. Excerpt of the Metamodel of the IND Knowledge Base

The different models not only use the same metamodel, but are also built in roughly the same way. We use conditions or norms (criteria) that tell whether a specific request is permitted or not. To establish this decision one or more characteristics of the foreigner (properties) are compared with legal grounds.

\subsection{Overall Architecture}

The architecture of the case systems with decision support is all about the separation of the knowledge from the process (separate the know from the flow). The case system is responsible for the data on files and persons, whereas the decision support system is responsible for the decisions taken based on (the correct) laws and legislation. The two components interact via web services with XML SOAP messages through an Enterprise Service Bus which only handles the transfer of messages.

The Case system uses web services to interact with the decision support system (knowledge as a service) for complex questions like: "Which activities need to be performed", "What is the risk concerning this applicant", "What decision should be made (automatically or supporting)", "Which publications should be made" (determine and assemble), calculate fees, etc.

The case system follows the generic reference process shown in figure 2 , that states that all processes of the Dutch Immigration Office are on some basic level the same. They all handle incoming requests, decide on the actions to be taken, process these actions and publish the results. Although the process is the same, the handling of a request never is. Each request has its own specific data, but the handling differs because of the knowledge applied. The relevant law and legislation relies on the data in the request, which for the Dutch Immigration Office is basically the purpose of stay. 


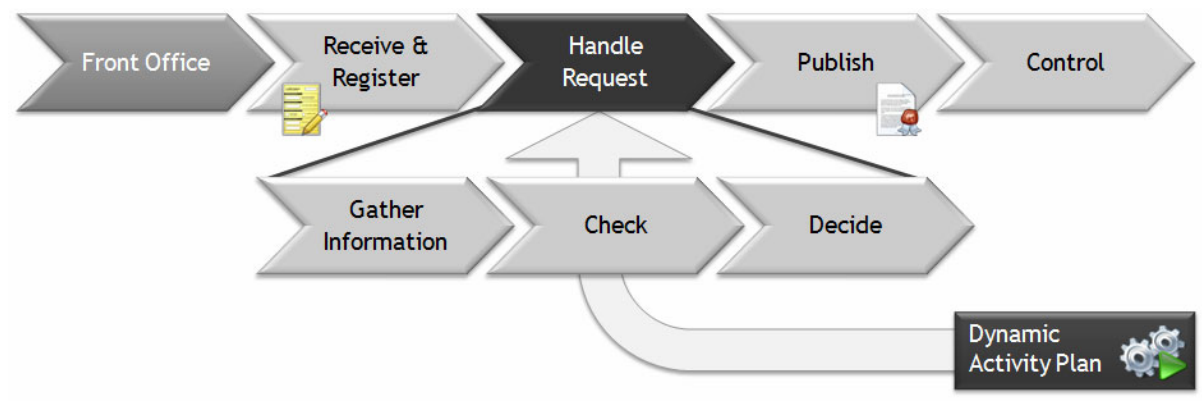

Fig. 2. Generic Reference Process to be filled by knowledge

The front office is built with SharePoint as the portal software and the decision support systems as the content supplier. This means (background) information, personalized advices, forms that enable submitted requests for permits and so on. This front office uses the same architectural knowledge backbone as the operational process. This means that they share the core taxonomies (the organizational vocabulary) mentioned before. This enables a seamless integration of the front office with the back office.

\subsection{Semantic Models as the Brain of the Infrastructure}

The knowledge workers request support on several domains in the operational process. This brought some key issues that the knowledge system had to solve:

1) Multilinguality; the application (especially the front office) needed to support multiple languages. Because $\mathrm{Be}$ Informed separates knowledge from presentation language specific labels are connected to the language independent models.

2) "Time travelling"; the knowledge models are implementations of laws and legislation, which are very dependent on time. We used timelines on (parts of) knowledge models to specify when they were relevant. Because all possible timelines are available in runtime this enables support for all cases, past, present and future, in the same operational process.

3) Skill based support; the knowledge workers involved in the process have different skills and authorization. Using typed textual references on models together with configuring user group related instruments, enabled us to show different users different (explanatory) texts.

4) Traceability \& compliance; each decision made by the decision support system needs to be traceable and compliant to laws and legislations. Partly this is covered by the software, but an important factor here is the Be Informed Methodology for Knowledge Maintenance[9]. Key in this methodology is that all models are built according to a metamodel. An overview of part of the IND metamodel is shown in figure 1 .

The decision support system uses the Model-Run-Analyze principle, meaning that the models (Model) are directly used in the operational process, there is no coding in between. This makes the model directly used in the day to day work (Run), which in its turn results into a direct feedback loop (Analyze). 


\section{Demonstration}

The audience will learn the importance of the separation of concerns and what this means in an operational process, and basically why this has won the Architecture Award 2009 in the Netherlands. The demonstration will give an overview of the architecture, but mainly focuses on the way decision support is integrated in the case system. The various levels of support for the knowledge workers, depending on authorization and skill level and the way decision support is used to guide the knowledge worker in the whole operational process.

We will show the metamodel including some examples of operational models, instruments and web services. This will be done using the model based catalog which is all about knowledge management and always in sync with the operational knowledge, because it uses the same knowledge models. Besides the architectural choices and implementation examples we will also demonstrate how we handled the mentioned knowledge related issues. Naturally, this will all be shown in the live application.

\section{References}

1. Antoniou, G.: A Semantic Web Primer, 2nd edn. The MIT Press, Cambridge (2008), ISBN-13: 978-026201242

2. Erl, T.: Service-Oriented Architecture (SOA): Concepts, Technology, and Design. Prentice Hall, Englewood Cliffs (2005), ISBN-13: 978-0131858589

3. Heller, R., van Teeseling, F.: Knowledge Applications for Life Events. In: The Semantic Web: Research and Applications: 6th European Semantic Web Conference, ESWC 2009, Heraklion, Crete, Greece, May 31-June 4 (2009)

4. IND, Dutch Immigration Office, http://www. ind.nl

5. IND Verblijfwijzer, http://www.verblijfwijzer.nl

6. Nederlands Architectuur Forum, http://www.naf.nl/nl/naf-prijzen/

7. OWL specification, http: / / www.w3.org/TR/owl-semantics /

8. RDF Specification, http://www.w3 .org/TR/rdf-concepts/

9. Van Teeseling, F., Heller, R.: Patterns in Metamodelling. In: Be Informed White paper 2008 (2008)

10. Van Teeseling, F., Heller, R.: Business Patterns in Ontology Design. In: Business Information Systems: 12th International Conference, BIS 2009, Poznan, Poland (2009)

11. Verbeek, J., Rensen, G.: A Citizen Centric Government Enabled by Semantic Technologies. In: Semantic Technology Conference, San Jose, CA, May 18-22 (2008) 